\title{
La République de Macédoine dans la presse française
}

La question du nom

Toni Glamcevski

\section{OpenEdition}

\section{Journals}

Édition électronique

URL : https://journals.openedition.org/ceb/822

DOI : $10.4000 / c e b .822$

ISSN : 2261-4184

\section{Éditeur}

INALCO

\section{Édition imprimée}

Date de publication : 30 mars 2011

Pagination : 197-212

ISBN : 978-2-85831-189-7

ISSN : 0290-7402

\section{Référence électronique}

Toni Glamcevski, «La République de Macédoine dans la presse française », Cahiers balkaniques [En ligne], 38-39 | 2011, mis en ligne le 05 décembre 2011, consulté le 06 juillet 2021. URL : http:// journals.openedition.org/ceb/822 ; DOI : https://doi.org/10.4000/ceb.822

Ce document a été généré automatiquement le 6 juillet 2021.

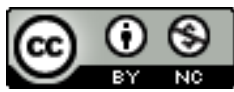

Cahiers balkaniques est mis à disposition selon les termes de la Licence Creative Commons Attribution - Pas d'Utilisation Commerciale 4.0 International. 


\section{La République de Macédoine dans la presse française}

La question du nom

Toni Glamcevski

"Imaginez-vous, un instant, vivant un affreux cauchemar. Vous voilà citoyen d'un pays à éclipses,

aux frontières caoutchouteuses, au territoire

protoplasmique entouré de voisins hostiles. La

"France ", dit l'Allemand? Ah oui, la Rhénanie occidentale? Mais non, rétorque l'Italien; vous savez

fort bien que ces « Français " sont des Piémontais francophones. Enfin, ceux du sud du pays. Au-dessus, ce sont des Gaulois ou des Germains, mais en aucun cas des Français, bien qu'ils s'entêtent à usurper ce nom.

Suffoqué, le Belge l'interrompt : pas du tout ! La "France ", c'est la Wallonie méridionale! Alors qu'ils en viennent aux mains, vous vous réveillez en sursaut, trempé de sueur et cour battant. Les «Macédoniens » - bien réveillés - vivent ce cauchemar-là depuis un siècle. Et aujourd'hui

encore..."

«Le Chaos Balkanique », Xavier Raufer, François

Haut

Malgré son nom illustre, la Macédoine demeure un des pays d'Europe les plus mal connus en France. Jusqu'en 1991, l'année de l'indépendance du pays, très peu de gens connaissaient l'existence de la République de Macédoine. Quand on évoquait la Macédoine, celle-ci était avant tout associée à l'Antiquité et à un mélange de fruits ou légumes. Au début du démantèlement de la fédération de l'ancienne Yougoslavie et de l'indépendance de la Macédoine, très peu d'informations à son sujet parvenaient en France. 
2 La Macédoine, longtemps restée en lisière des conflits yougoslaves, excepté durant l'année 2001, n'a pas attiré un grand intérêt dans les médias français. Quand on écrivait à son sujet, c'était souvent dans le contexte de conflits voisins, particulièrement pendant la guerre du Kosovo. Les médias français commencent à s'intéresser vraiment au pays lors du blocage de sa reconnaissance par la Grèce (1991). De nombreux articles, commentaires, reportages, interviews et rappels historiques, tentent alors d'éclaircir la situation du pays, mais souvent avec de médiocres connaissances sur celui-ci et la région en général.

3 Dans la période qui va du printemps 1999 au 11 septembre 2001, il y eut une intense production journalistique au sujet de la Macédoine, tant dans les journaux que dans les services de communication des institutions et organisations internationales et nationales. Et, dans ce cas, ce qui est une exception sur le traitement médiatique des conflits de l'ex-Yougoslavie, il y a eu très peu d'interventions de la part des intellectuels et experts.

4 Cet exposé a pour but d'apporter des éclairages sur le traitement médiatique du conflit qui a opposé la République de Macédoine à la Grèce dans la presse française de 1991 à aujourd'hui, et sur certains aspects de la question du «nom ». Ce n'est pas une analyse du travail journalistique, mais de l'image que les médias ont donné de la Macédoine à travers la presse française, sachant que les journalistes écrivent souvent la première version de l'histoire et influencent l'opinion publique. ${ }^{1}$

5 La période entre 1991 et 2009 peut être facilement partagée en deux parties : la première - l'indépendance du pays et sa lutte pour la reconnaissance internationale ; la deuxième - la Macédoine pendant le conflit du Kosovo : le conflit de 2001 sur son territoire et la Macédoine post conflictuelle, en d'autres termes les relations entre les Macédoniens et les Albanais.

\section{De 1991 à 1998}

« Diplomatiquement, la question de la Macédoine est tranchée, la Macédoine aussi, en trois morceaux, le serbe, le grec et le bulgare. Le traité de Neuilly a recueilli, à ce sujet, toutes les signatures nécessaires. $»^{2}$ - écrivait, en 1931, Albert Londres.

7 Les articles sur la "poudrière des Balkans", écrits 60 ans auparavant par Albert Londres, sont encore d'actualité. Il reconnaît implicitement, en 1931 que « la question de la Macédoine » n'est pas réglée, en 2009 elle ne l'est pas non plus, bien que tranchée.

Les premiers articles sérieux sur la Macédoine ont été publiés courant 1991, plus précisément après le référendum du 8 septembre. La presse parisienne va titrer : «Le oui à l'indépendance de la Macédoine », un vote de survie pour le plus pauvre des États yougoslaves. Libération (lundi 9 septembre 1991) rappelle qu'« officiellement il n'est pas question de sécession. Les électeurs de Macédoine ont été priés de répondre à la question de savoir s'ils veulent oui ou non, vivre dans une république souveraine et indépendante dotée du droit à une association d'États yougoslaves souverains. » Si la Macédoine entend marcher vers l'indépendance en toute prudence, c'est bien parce que ses dirigeants sont conscients que cette république vit dans un environnement particulièrement hostile, où Bulgares et Serbes cherchent à se l'approprier, où les Grecs craignent de voir ressusciter sur leur sol la question macédonienne, et où les Albanais visent la partie ouest, écrit ce journal. 
«Les Macédoniens ont massivement participé au référendum sur l'indépendance de leur République.» En votant ainsi, "La Macédoine entend se prémunir contre un éventuel échec des négociations sur l'avenir de la Yougoslavie, qui la laisserait dans une fédération amputée de la Slovénie et la Croatie, mais dominée par la Serbie ", conclut Le Monde (mardi 10 septembre 1991).

10 Les difficultés résident dans la reconnaissance de la Macédoine en tant qu'État indépendant, dans les relations avec la Grèce ${ }^{3}$. De nombreux articles rendent compte des efforts de la Macédoine pour sa reconnaissance internationale. Le Monde expose l'attitude de la France face à la reconnaissance des Républiques ex-yougoslaves. La reconnaissance se fera "avec des nuances et des délais", selon M. Roland Dumas, ministre des Affaires étrangères. "Sur les quatre » Républiques qui ont sollicité cette reconnaissance « on pourra déjà en reconnaître quelqu'une, peut-être, pour les autres, le fera-t-on de façon différée dans le temps ». La Macédoine doit encore attendre, selon Libéation (mercredi 15 janvier 1992), alors que Le Monde (jeudi 16 janvier 1992) annonce « Oui » à la Slovénie, « oui » à la Macédoine, «non » à la Bosnie-Herzégovine ». « Quant à la Croatie, elle est priée de revoir un peu sa copie... », telles sont les conclusions de la commission d'arbitrage, composée de cinq membres des cours ou tribunaux constitutionnels européens et dirigée par M. Robert Badinter ${ }^{4}$

11 Libération (mercredi 15 janvier 1992) et Le Monde (jeudi 16 janvier 1992) transmettent la déclaration du président François Mitterrand donnée à l'issue de sa visite à Luxembourg dans laquelle il exclut une reconnaissance immédiate de la Macédoine :

"C'est une question prématurée. Là, peut-être plus encore qu'en Croatie se trouve posé le maximum de conséquences d'ordre international. La France ne s'engagera pas légèrement dans cette affaire et pour l'instant ne recommande pas du tout cette reconnaissance. [...] Nous n'avons pas reçu de demande officielle de la République yougoslave de Macédoine, je n'ai donc pas à répondre à cette question.»

12 La journaliste du Monde, Claire Tréan rappelle que le président Mitterrand a oublié que les autorités de Skopje avaient formulé cette requête en décembre 1992, et conclut «M. Mitterrand ne s'en remettra donc pas, à propos de la Macédoine, à l'avis des juristes.»

13 Cela relativise quelque peu la portée de la méthode que la France avait elle-même préconisée à ses partenaires européens, face aux demandes de reconnaissance de nouveaux États : établir une série de conditions auxquelles toute entité aspirant à l'indépendance doit obéir et, dans le cas des Républiques yougoslaves, demander à la commission d'arbitrage d'évaluer si ces conditions sont effectivement remplies ». La position française est longtemps restée incomprise par l'opinion publique macédonienne et, indirectement a contribué à la déstabilisation du pays, la laissant sans reconnaissance internationale.

14 Le Monde (mardi 28 janvier 1992) publie l'article de son correspondant d'Athènes, Didier Kunz, "La Macédoine poudrière des Balkans », qui essaye de donner une analyse du " conflit»; cet article est le miroir de la position officielle de la diplomatie grecque et de ceux qui nient l'existence du peuple macédonien. Dans le texte on lit entre autres : «...Maréchal Tito, d'origine croate, a créé, en 1945, la Macédoine comme État-tampon afin de limiter la puissance de la Serbie vers le sud... » Ici nous avons le parfait exemple de la méconnaissance de l'histoire qui peut induire en erreur les lecteurs. Le journaliste ignore que la République de Macédoine a été déclarée, le 2 août 1944, par les délégués du peuple macédonien de l'Assemblée antifasciste pour la libération populaire de la 
Macédoine (ASNOM), et non par Tito. La minorité macédonienne en Grèce sous la plume du journaliste se transforme en population bulgare, citant un rapport de la Société des Nations de 1926, selon lequel la Macédoine grecque avait établi à l'époque une unité ethnique composée de $89 \%$ de Grecs et de $5 \%$ de Bulgares. Donc, les Macédoniens n'existent pas. Il oublie de rappeler que, la même année, le gouvernement grec, par décret, change tous les toponymes de la Macédoine grecque en les hellénisant, menant une politique d'assimilation forcée de la population non grecque ${ }^{5}$.

M. Kunz fait partie des premiers journalistes qui utilisent le terme «slave" pour qualifier le peuple macédonien. Dans son article publié dans Le Monde (mardi 28 janvier 1992) il représente l'Organisation révolutionnaire macédonienne intérieure (VMRO, ou ORIM), comme une organisation bulgare. Selon lui «des intellectuels bulgares créent notamment l'Organisation révolutionnaire macédonienne intérieure (VMRO, ou ORIM), qui donnera naissance aux fameux « comitadjis $»^{6}$ dont les actions terroristes sont décrites, en 1931 par Albert Londres... ».

RFI programme en langue serbo-croate (mercredi 29 janvier 1992) répond à cette analyse du Monde avec une sévère critique, signée par la journaliste Maria Bezanovska. Une grande partie de cette critique est reproduite quinze jours plus tard dans Le Quotidien de Paris (vendredi 14 février 1992) sous le titre "Au nom de la Macédoine ", dans lequel elle répond avec ses arguments aux arguments des Grecs, en faisant un rappel historique (qui n'est jamais facile à faire quand il s'agit des Balkans).

À partir du samedi 4 avril 1992, Le Monde commence à publier une série d'articles d'Yves Heller consacrés aux relations entre la Macédoine et la Grèce. La première page titre "Passions macédoniennes", un grave conflit politicosémantique oppose Athènes et Skopje. Dans le sous-titre le journal évite d'utiliser le nom du pays, mais, dans le grand reportage, il montre d'une manière impartiale le climat régnant en Grèce : « Un climat, d'abord, où le passionnel prime devant ce véritable «crime " commis par les gens de Skopje - il n'est pas question de prononcer le mot même de Macédoine lorsque l'on parle de la République ex-yougoslave -- quand ils ont décidé à l'instar des Slovènes et des Croates de déclarer unilatéralement leur indépendance en conservant l'appellation sous laquelle leur République est connue depuis quarante-cinq ans, à savoir République de Macédoine.» C'est une parfaite illustration d'un nationalisme nourri par des politiques, médias et scientifiques (des archéologues) et rumeurs, comme celle qui accuse le "Vatican d'avoir payé les Pays-Bas pour que ceux-ci reconnaissent " la République de Macédoine. Toujours selon ses dires, «le boycottage des produits néerlandais et allemands organisé en Grèce a empêché cette reconnaissance ». Pour mener à bien ses noirs desseins, le pape agit dans l'ombre : « Derrière Gligorov, il y a De Michelis et, derrière De Michelis, il y a le pape.», «Ce que fait le pape est un grand danger ».

Trois jours plus tard, Le Monde (mardi 7 avril 1992) publie un nouvel article, mais cette fois de Skopje avec pour titre «La reconnaissance de la Macédoine est devenue une question d'éthique pour la CEE»", citant les paroles du président Gligorov. Le président macédonien présente la position de son pays, rassurant son voisin du sud en lui assurant qu'il n'a pas de prétentions territoriale et historique. Gligorov explique ici qu'il ne voit pas ce que ça apportera à la Grèce de changer le nom de Macédoine et ce qu'elle perdra « si nous gardions le nom de Macédoine. Nous habitons une partie de la région que l'on appelle la Macédoine, et cela fait des siècles que nous nous appelons Macédoniens. Cela n'a, bien sûr, aucun rapport avec les Macédoniens de l'époque 
d'Alexandre le Grand. Nous sommes slaves, venus ici au cours des sixième et septième siècles, et, pour nous différencier des autres peuples slaves, des Bulgares, ou des Serbes, nous avons pris le nom du territoire sur laquelle nous nous sommes installés... ».

Cette déclaration du président macédonien a eu de lourdes conséquences plus tard, contribuant directement à l'utilisation du terme « slaves » pour distinguer, le peuple, la langue ou la culture macédonienne des autres groupes ethniques vivants en Macédoine et dans les Balkans, et montre aussi aux Grecs que « la Macédoine est prête à faire des concessions ".

Parlant de la décision des Douze de reconnaître la Bosnie-Herzégovine, alors que les combats se poursuivent toujours dans cette République, Libération (mardi 7 avril 1992) constate que « la Macédoine, elle, devra encore attendre sa reconnaissance - "l'onction des Douze"bloquée par les Grecs ». Le Monde (mardi 30 juin 1992) publie l'intégralité de la déclaration des Douze sur l'ex-Yougoslavie, après le conseil européen de Lisbonne. Une déclaration qui va complètement dans le sens de la Grèce, renforçant sa position vis-à-vis de la Macédoine.

21 "La Communauté et ses États membres se déclarent "disposés à reconnaître cette République dans ses frontières actuelles conformément à la déclaration du 16 décembre 1991, sous une dénomination qui ne comporte pas le terme Macédoine" ! ॥ Une décision sans précédent dans le droit international, qui aura de lourdes conséquences politiques et économiques pour la République de Macédoine.

La presse française défend sans exagérer « la cause macédonienne » dans le conflit qui l'oppose à la Grèce. On lit les titres suivants : «La scène se passe en Macédoine, c'est-àdire nulle part $»^{7}$, «La Communauté face aux querelles byzantines $»^{8}$. Sous le titre «Léonidas réveille-toi!», l'hebdomadaire Le Nouvel Observateur, constate que «la proclamation d'indépendance de l'ancienne République yougoslave de Macédoine a provoqué le déferlement, dans toute la Grèce, d'une vague de nationalisme "*. "Les Grecs font le forcing contre la Macédoine " écrit Libération (vendredi 11 décembre 1992), en parlant de la «foule immense (de l'ordre de 1,3 millions personnes), répondant à l'appel des autorités, a manifesté, hier à Athènes, pour faire entendre aux Douze que la reconnaissance de l'ex-République yougoslave au sommet d'Édimbourg serait très mal venue ».

Le Monde (samedi 12 décembre 1992) rappelle que la Macédoine intéresse ses autres voisins: "Macédoine, le rêve perdu des Bulgares" et raconte comment Sofia, sagement, revoit ses positions sur la pomme de discorde des Balkans ».

Dans ce contexte politicoéconomique suivent nombre d'articles sur la nonreconnaissance du pays. "Qui a peur de la Macédoine ${ }^{10}$, "Macédoine: la Grèce isolée » lisait-on dans l'éditorial du Monde ${ }^{11}$.

25 Au lendemain de l'enregistrement de la demande d'adhésion de la Macédoine à l'ONU, et alors que les consultations sont engagées sur la candidature, les dirigeants de la Macédoine, réaffirment leur volonté de ne pas céder aux pressions grecques, pour changer le nom, ce qui amène Le Monde (vendredi 29 janvier 1993) à écrire «La Macédoine veut rester la Macédoine ", "Inquiétude macédonienne »" où l'envoyé spécial conclut que «Plus que la Grèce, les autorités de Skopje craignent l'activisme des minorités albanaise et serbe ». (12 mars 1993). Selon lui « il semble exister une réelle conscience macédonienne, qui au 
même titre que pour les Palestiniens suffit à elle seule à légitimer la revendication d'un État. Les Grecs, qui au fond n'opposent pas d'obstacle à la reconnaissance de cet État en échange d'un changement de dénomination, n'ont pas, à cet égard, fait preuve de beaucoup de compréhension, pas plus qu'ils n'auront pas réussi à se faire comprendre. » Dans son article M. Nicoladis, a ouvert une piste de réflexion sur le modèle de l'État macédonien : État-nation ou État territoire, qui aura sa réponse huit ans après avec les Accords d'Ohrid.

Simultanément sont publiées dans la presse française plusieurs tribunes, opinions, polémiques et analyses consacrées à la Macédoine et à sa reconnaissance internationale. Comme celle de Nicolas Marcos, «La Macédoine est-elle grecque depuis quatre mille ans?", dans la revue L'Histoire ${ }^{13}$. Dans les conclusions de son article, Nicolas Marcos exprime son opinion sur la reconnaissance de la République de Macédoine. «En refusant que la Macédoine yougoslave devienne indépendante sous son nom, la position du gouvernement grec est, on le voit, intenable. Le terme de Macédoine a désigné dans l'histoire des réalités ethniques très variables, souvent non helléniques, et il n'est pas plus la propriété de la Grèce que le mot «France » serait la propriété de l'Allemagne parce qu'il serait issu d'une peuplade germanique. La proposition grecque de rebaptiser la Macédoine yougoslave «Dardania » (du nom d'un peuple antique mal connu) ou "République du Vardar» a autant de sens que si l'on appelait désormais la Grande-Bretagne "Cassitérie » ou la France « République de Seine et Rhône ».

Selon l'auteur de cet article, "les grandes puissances ont aussi leur part de responsabilité. En attendant, historiens et archéologues étrangers ont été sommés par leurs collègues grecs de signer des proclamations quant aux droits de la Grèce sur la Macédoine. D'autres se sont vu refuser des communications scientifiques sur la présence des Slaves en Grèce à l'époque médiévale. Certes, la manipulation de l'histoire est une vieille tradition balkanique - qu'il s'agisse de la glorification d'une antiquité illustre largement dévoyée ou de l'amnésie sur les cinq siècles d'occupation ottomane au prix de la destruction de tous les monuments de cette période - mais de tels excès sont difficilement acceptables de la part d'une Grèce où s'enracinent bien des mythes fondateurs de notre actuel Occident $» .^{14}$

Cet article est suivi d'un autre, intitulé : «La Macédoine est-elle Grecque ? $^{15}$ ». Deux points de vue opposés sont présentés par L'Histoire. Celui de Pierre Coavoux, professeur de lettres classiques et chargé de cours en civilisation grecque à l'université de Lyon-III, qui n'accepte pas les positions de Nicolas Marcos qui déplorait qu'aujourd'hui en Grèce les passions nationalistes l'emportent sur l'analyse objective des faits : on y revendique la Macédoine au nom d'une histoire largement falsifiée. Il justifie la position grecque, estime que les positions les plus radicales sont l'œuvre de groupuscules extrémistes.

Dans la réponse, Nicolas Marcos, défend de nouveau ses positions points de vue, écrivant que «la lettre de Pierre Coavoux a le mérite de réaffirmer les principales thèses grecques. " «Les slogans "La Macédoine est grecque depuis 4000 ans" ou "depuis toujours", "La Macédoine, c'est les Thermopyles de l'hellénisme" et bien d'autres, n'émanent pas de "groupuscules extrémistes" mais sont très officiellement placardés dans les aéroports et aux frontières", argumente Nicolas Marcos. "Le nom de la Macédoine yougoslave n'est pas une manipulation récente de la propagande communiste: les populations de cette région se nomment elles-mêmes “macédoniennes" depuis longtemps. ». N. Marcos est étonné par l'absence de données 
grecques sur l'effectif de la minorité macédonienne de Grèce. « Assurément, la Grèce ne répondrait pas en terme de droits des minorités aux conditions que l'on exige désormais, pour les reconnaître, des nouvelles nations... ». "Si la Macédoine n'est pas grecque depuis 4000 ans, la Grèce (antique) est pourtant la patrie de la démocratie... », conclut Nicolas Marcos dans L'Histoire.

Le Monde du 9 avril 1993 consacre son éditorial à l'adhésion de la Macédoine à l'ONU : «Pour la première fois de son histoire, l'organisation internationale reconnaît un État sans nom ni drapeau, c'est-à-dire sans les attributs minimaux de la souveraineté. Mais la Macédoine a quand même de la chance dans son malheur, car elle est passée du stade du non-être à celui d'une existence semi-clandestine ».

Le 17 décembre 1993, on enregistre les premières réactions après l'établissement des relations diplomatiques et la reconnaissance de la Macédoine par six pays de l'Union européenne, dont la France. Le Figaro titre "La Macédoine: la Grèce se dit trahie ", Libération: «La Macédoine reconnue sous son autre nom», Le Monde (samedi 18 décembre 1993) consacre son éditorial à cette affaire: "Le nœud gordien macédonien ».

Aujourd'hui, le problème avec la Grèce sur le nom de la République de Macédoine n'est pas encore résolu devant l'ONU. La presse française, à la différence du début de ce conflit sémanticohistorique, reste très factuelle.

\section{Le Nom du Peuple/Nation.}

Dans la presse française et la presse occidentale en général, avec l'assimilation du peuple macédonien au simple terme de "Slaves ", on touche directement à la question de l'identité macédonienne. Les médias, surtout pendant le conflit du Kosovo et le conflit de 2001 sur le territoire de la Macédoine, voulaient sans doute éclaircir la situation, quand ils écrivaient ou parlaient des Macédoniens en les appelant simplement "Slaves». Mais l'utilisation de ce terme pourrait aussi être comprise comme une tentative de négation de l'existence du peuple macédonien.

On pourrait citer de nombreux exemples, comme Le Figaro du 14-15 juillet 2001 qui écrivait: "Les Occidentaux poussent Slaves et Albanais à s'entendre ", Le Monde du samedi 11 août 2001 «L'escalade des combats en Macédoine compromet l'accord de paix entre Albanais et Slaves ». Paradoxalement, dans le même article, dans un encadré, on parle des obsèques de dix Macédoniens tués dans des gorges entre Tetovo et Skopje.

Dans leurs travaux, certains journalistes par ignorance, par simplification ou dans le souci d'être plus clair, ou encore sciemment, d'autres comme Alexandre Adler dans Le Courrier international, ont ignoré la vérité et les faits, ont nié l'existence de l'identité de la langue et de la nation (peuple) macédonien(ne). Dans ses blocs-notes «Pour les Balkans, Chirurgie ou homéopathie ?» (L'éditorialiste) Alexandre Adler dans Le Courrier international ( ${ }^{\circ} 545$ du 12 au 18 avril 2001), va plus loin en assimilant les Macédoniens aux Bulgares, « ... tandis que les Slaves macédoniens (en réalité bulgaro-slaves) de la région de Tetovo se replieraient vers l'est $»$. Avec un simple coup de plume, il nie l'existence et l'identité d'environ trois millions d'êtres humains, qui se définissent euxmêmes comme Macédoniens.

On peut dire seulement que cet usage du terme «Slave " pour Macédoniens est devenu presque courant dès le début des hostilités au Kosovo en 1999. Nous avons été témoins 
alors de sérieuses erreurs dans la presse, particulièrement quand il s'agissait de parler des Macédoniens, à cause de l'ambiguïté de l'utilisation de ce terme, évoqué parfois de façon injustifiée. Dans un reportage du journal télévisé sur France 3, on a entendu « La population majoritaire du pays, ce sont des Slaves, que les Albanais appellent "Macédoniens "...alors que l'individu interrogé, pour les besoins du reportage, parlait clairement de Macédoniens, et non de "Slaves ». Dans un autre reportage de France 3, le journaliste disait que «la majorité de la police et de l'armée macédoniennes sont constituées de Serbes »! Les erreurs volontaires ou involontaires peuvent introduire une confusion auprès du public. Les Macédoniens ont eu le sentiment que l'on niait leur identité.

\section{Le peuple macédonien et l'identité macédonienne sont une réalité} une « appellation » qui la relie au passé l'empêchant d'avancer. C'est une désignation provisoire créée en 1993 à l'usage spécifique des Nations Unies, dans l'attente d'un règlement du différend qui a surgi en raison du nom constitutionnel de «République de Macédoine» que ce pays s'est choisi lorsqu'il est devenu indépendant. Cette désignation provisoire, initialement prévue à l'usage des Nations Unies, a ensuite été adoptée par d'autres organisations internationales, notamment le Conseil de l'Europe, 
l'Union européenne et l'OSCE. Ce différend oppose «l'ex-République yougoslave de Macédoine » à la Grèce.

À ma connaissance, c'est la première fois dans l'histoire que le choix d'un nom a créé un différend bilatéral. Les seuls exemples en Europe qui me viennent à l'esprit susceptibles d'avoir pu créer une situation similaire sont ceux du Luxembourg, qui est à la fois le nom d'un État souverain et le nom d'une province de Belgique et celui de la Moldova, qui est à la fois un État souverain et une région de la Roumanie. Les similitudes s'arrêtent cependant là, car, pour autant que je sache, il n'y a pas de rivalité politique quant à l'utilisation de ces noms.

Le conflit entre la Grèce et la République de Macédoine est en revanche très passionné et vécu de manière intense par les populations des deux pays. Il dure aussi depuis longtemps : plus de quinze ans.

La question du nom est souvent banalisée, minimisée et mal comprise. Il est possible également que la longueur de la controverse ait engendré une certaine lassitude pour ceux qui ne sont pas directement concernés, ayant comme résultat que les motivations des deux parties et l'état des négociations ne sont pas totalement compris. Je suis sûr qu'ils sont peu nombreux à avoir réalisé que la Grèce est prête à accepter le nom "Macédoine ", mais cela uniquement dans le cadre d'un nom composé ; de même, ils ne sont pas nombreux à savoir que le nom constitutionnel a été reconnu sur le plan bilatéral par 115 États, dont un nombre limité en Europe ; encore moins sont informés que les parties n'ont pas le même avis sur la portée des négociations.

\section{Rapprochement vers l'UE conditionné par le nom}

46 L'échec de l'adhésion à l'OTAN est une grande déception, bien que la porte reste ouverte en cas de compromis sur le nom. La situation est la même concernant l'adhésion à l'UE. Le Parlement européen a déclaré que le nom ne devait pas représenter un obstacle à l'adhésion de la Macédoine dans les autres institutions internationales. Le Parlement européen est d'avis que de telles questions bilatérales en suspens dans les Balkans ne devraient pas faire obstacle à l'adhésion ou prendre le pas sur le processus d'intégration européenne. ${ }^{16}$

Le nom de la République de Macédoine est devenu une condition pour son intégration euro atlantique! L'intégration européenne constitue un objectif fédérateur dans un pays où les populations macédonienne et albanaise sont loin de s'accorder sur tout. Cette perspective stimule les réformes et renforce la confiance des investisseurs.

En l'absence de compromis sur le nom, le gouvernement grec a déjà annoncé qu'il s'opposerait à cette adhésion, peut-être dès cette année, à un moment où la Macédoine est dans l'attente depuis 2005 d'une date pour l'ouverture des négociations.

La République de Macédoine dans la querelle qui l'oppose à la Grèce considère qu'elle est la seule à avoir fait des efforts : elle a modifié son drapeau et sa constitution, a renoncé à toute prétention territoriale vis-à-vis de la Grèce, n'a jamais provoqué d'incident frontalier. Elle adopte donc un comportement tout à fait normal, voire exemplaire dans la région. La Grèce de son côté n'a fait comme seule concession que l'acceptation du nom "Macédoine» dans le nom composé de la République de Macédoine. Mais les négociations menées par le médiateur de l'ONU, Matthew Nimetz, ne progressent pas. 
50 L'Union européenne semble toujours solidaire de la Grèce. Celle-ci dispose du droit de veto, et les autres pays ne peuvent changer sa décision. À ce titre, la récente déclaration de soutien à la Grèce du président français M. Nicolas Sarkozy a fait débat. Elle fut ensuite nuancée, la France défendant une " position acceptable » pour les deux pays. Pour finalement affirmer de nouveau son soutien à la position grecque

Imposer à un pays de changer de nom suite à la contestation par un pays voisin de la légitimité du nom constitue un précédent dans le droit international. Tout État devrait pouvoir choisir son nom librement! Au-delà de la question du nom, beaucoup d'autres questions plus compliquées se posent, dans un contexte régional difficile, il s'agit :

- du concept de nation, de minorité nationale et de leur application ;

- de la montée du nationalisme ;

- de l'interprétation et de la manipulation de l'histoire à des fins politiques ;

- de l'enseignement de l'histoire.

Peut-être devrait-on se pencher davantage sur ces questions.

Aujourd'hui, peut-être plus que jamais, il est nécessaire de définir ce qu'«être Macédonien » veut dire, dans le cadre des changements intervenus avec l'Accord-cadre d'Ohrid. Mais cette tâche incombe aux Macédoniens et non pas à ses voisins et à l'Union européenne.

\section{NOTES}

1. Lorsque les journalistes et les médias relatent les événements, dans une certaine mesure ils écrivent l'histoire, car les travaux des historiens vont dépendre de leurs écrits. S'ils se trompent, les historiens se tromperont aussi. Les gens et les chercheurs aussi croient de plus en plus en ce qu'ils lisent dans les journaux et travaillent sur la presse. Les journalistes écrivent finalement la première version de l'Histoire. Le journaliste et historien Timothy Garton Ash (Interview dans Libération 6-7 octobre 2001) est convaincu d'ailleurs qu'« en réalité, il n'y a pas de claires lignes de partage entre ce que font un chercheur en histoire contemporaine et un bon journaliste. Tous deux utilisent des sources communes et ont recours aux mêmes instruments intellectuels, mais ce sont des professions différentes ».

Le premier compte rendu du passé aujourd'hui est le reportage. Les souvenirs des participants divergent souvent; le journaliste, à la différence du participant qui donne sa version des faits, tente, lui, de trouver ce qui est réellement arrivé. C'est l'essence de son travail. Le problème des spécialistes de l'histoire de l'Antiquité est qu'ils n'ont pas assez de sources. Ils sont obligés de reconstruire toute une civilisation à partir de fragments. Aujourd'hui, nous disposons par contre de trop de matériel. L'Histoire est rapportée vingt-quatre heures sur vingt-quatre, et en temps réel, à la télévision, à la radio, sur Internet et tous les jours dans la presse écrite. Si nous voulons écrire l'histoire d'un pays, qui a été touché par un conflit largement couvert par les médias, nous aurons besoin d'autant de temps pour visionner ou lire les récits. Ici se pose le problème de sélection.

2. Albert Londres, Les Comitadjis, Le Serpent à Plumes, 1997, p. 187.

3. Déclaration de la CEE sur la Yougoslavie 16 décembre 1991. Les ministres des Affaires étrangères des États membres de la Communauté européenne adoptaient, à Bruxelles, une 
Déclaration sur la Yougoslavie. Cette Déclaration disait entre autres que les demandes des Républiques ex-yougoslaves qui souhaitent être reconnues «seront soumises par la présidence de la Conférence européenne à une Commission d'arbitrage ». Ainsi, une des dispositions de la Déclaration demandait qu' « une République yougoslave [...] s'engage, avant qu'elle soit reconnue, à donner des garanties constitutionnelles et politiques assurant qu'elle n'a aucune revendication territoriale vis-à-vis d'un pays voisin membre de la Communauté et à ne pas conduire d'activités hostiles de propagande contre (ce même pays), y compris l'utilisation d'une dénomination impliquant des revendications territoriales». Par la suite, en dépit d'un avis favorable de la Commission d'arbitrage qui suivait l'amendement de la Constitution macédonienne en vue de se conformer aux recommandations de la Déclaration de Bruxelles, la Macédoine ne fut pas reconnue par les États de la Communauté européenne en raison des fortes réserves exprimées par la Grèce.

4. La Macédoine pouvait s'attendre à être reconnue par les Douze, elle présente "peu de problèmes ». La commission Badinter note que, "les membres des diverses nationalités (dont, par conséquent, la minorité albanaise de souche) ont le droit d'exprimer librement leur identité nationale, de la cultiver, en poursuivre le développement, et ont droit également à leurs attributs nationaux». La Constitution de la République souligne également que la Macédoine "n'a aucune revendication territoriale à l'égard des États voisins » et "s'interdit toute propagande hostile à l'encontre d'un autre État ». Mais ces affirmations « sans ambiguité" ne vont pas du tout calmer le gouvernement d'Athènes qui s'oppose systémiquement à ce qu'un pays porte le nom de « République de Macédoine », celle-ci étant de son point de vue la région du nord de la Grèce.

5. En 1925, la Grèce est obligée au terme du Traité de Sèvres de faire imprimer un abécédaire «l'ABECEDAR » dans le dialecte de Florina et de Bitola en caractères latins. Les réajustements internationaux, les mécanismes « autonomes » locaux et la réaction de la Serbie et de la Bulgarie font que celui-ci tombe en désuétude. Lors de la Conférence balkanique de 1929, la partie grecque songe de nouveau à introduire la langue "slavo-macédonienne " à l'école. En 1944, dans les régions de Macédoine occidentale contrôlées par les rebelles de gauche, le SNOF édite un abécédaire établi d'après les variations dialectales parlées à Florina, Kastoria et Bitola, en alphabet cyrillique. Par la suite, la langue commence à être enseignée dans les départements de Florina et de Kastoria, et un effort est entrepris pour former des maîtres. Les réfugiés de gauche, dans les pays de l'Est, éditent un abécédaire, sinon plusieurs, en 1950 à Bucarest, un autre en 1966 en Pologne, ainsi que des manuels scolaires où des rudiments de la langue sont enseignés.

Patrick Seriot à propos de l'utilisation de la langue macédonienne en Grèce à écrit : "Mention spéciale doit être faite des "serments de langue", marque extrême de l'hellénisation forcée des slavophones de Grèce. Dans certains cas, un serment collectif public a été imposé aux habitants de villages slavophones, stipulant qu'ils juraient de ne plus jamais parler slave, mais grec, même chez eux ». Ces événements se sont déroulés durant l'été 1959, ils sont rapportés par Pribišević (1982, p. 245), qui cite des journaux grecs de l'époque. Le serment disait ceci :

«Devant Dieu et les hommes, en tant que fidèles descendants des anciens Grecs, nous jurons qu'à l'avenir nous n'utiliserons plus, nulle part, et jamais, le dialecte slave ». - dans « Faut-il que les langues aient un nom ? Le cas du macédonien », in Andrée Tabouret-Keller (éd.) : Le nom des langues. L'enjeu de la nomination des langues, vol. 1, Louvain : Peeters, 1997, pp. 167-190

6. L'utilisation du mot "comitadjis", d'Albert Londres avait un caractère péjoratif, moqueur, outrager pour minimiser la lutte du peuple macédonien, et servait la propagande pour la Grande Serbie, écrit Manol Pandevski, membre de l'Académie macédonienne, dans l'avant-propos de l'édition macédonienne du livre « Comitadjis".

7. Libération, 12 mai 199 : le journal rappelle que les Albanais de Macédoine ont ainsi proclamé la création, sur la partie du territoire macédonien où ils sont majoritaires, de la «République autonome d'Illyrie ». Libération ne donne guère plus d'informations sur les revendications et le 
nombre des Albanais de Macédoine, mais en parlant de ce recensement, le journal donne un avant-goût de la polémique qui va être également une des sources du conflit dans ce pays.

8. Le Monde, mardi 26 mai 1992.

9. Le Nouvel Observateur, 1-7 octobre 1992 - «Ils étaient les symboles de la Grèce démocratique, de la Grèce libre, de la Grèce tolérante. À l'époque du film « $\mathrm{Z}$ » et des colonels d'Athènes, ils ont fait vibrer toute la gauche européenne. Aujourd'hui, Mélina Mercuri et Mikis Théodirakis sont engagés dans un combat plus douteux. L'actrice, ancienne ministre socialiste de la Culture, et le compositeur longtemps emprisonné par la dictature, celui qui a composé l'hymne du PS français, figurent parmi les artisans les plus actifs de la flambée nationaliste qui secoue la Grèce. ».

10. L'Express, 14 janvier 1993.

11. Le Monde, vendredi 22 janvier 1993 « Le président de la CEE, le ministre danois des Affaires étrangères président en exercice du conseil des ministres de la CEE, M. Uffe Elleman-Jensen a usé et abusé (selon le correspondant du Monde de Strasbourg), mercredi 20 janvier, devant les députés européens, de sa situation singulière. Jouant constamment sur le fait qu'il avait de fortes chances de ne plus être ministre au lendemain de son intervention, il a pratiqué l'humour à haute dose. "Je suivrai à la télévision la prestation de mon successeur lors du conseil des ministres des Affaires étrangères des Douze qui se tiendra le $1^{\text {er }}$ février prochain à Bruxelles. Le gouvernement grec peut demander ma démission ", - a-t-il déclaré après avoir déclenché la colère des parlementaires grecs en disant : "J'ai honte dans l'affaire de la Macédoine de l'attitude de la Grèce qui se sent menacée par un tout petit pays faible alors qu'elle est assurée de la solidarité de ses partenaires de l'OTAN.» Loin de s'excuser, comme le lui demandaient les représentants d'Athènes, il a enfoncé le clou : « La Communauté ne peut pas se laisser prendre en otage par nos amis grecs qui, sur ce dossier, ne font pas preuve d'esprit communautaire. » Pour couronner le tout, M. Elleman-Jensen a formulé le vœu, que le Conseil de sécurité de l'ONU décide rapidement de sorte que les « Onze » puissent - Danemark en tête - établir des relations diplomatiques avec le nouvel État ».

12. Le Monde, vendredi 12 février 1993.

13. L'Histoire, $\mathrm{n}^{\circ} 160$ novembre 1992.

14. L'Histoire, $\mathrm{n}^{\circ} 160$ novembre 1992.

15. L'Histoire, $\mathrm{n}^{\circ} 163$ février 1993.

16. P6_TA-PROV (2009) 0135, Rapport de suivi 2008 sur l'ancienne République yougoslave de Macédoine - Résolution du Parlement européen du 12 mars 2009 sur le rapport de suivi 2008 concernant l'ancienne République yougoslave de Macédoine.

\section{RÉSUMÉS}

Les médias français s'intéressent à la République de Macédoine quand la Grèce refuse de la reconnaître, mais souvent adoptent des positions progrecques.

Malgré son nom illustre, la Macédoine est un pays très mal connu en France. Jusqu'en 1991, très peu de gens connaissaient l'existence de la République de Macédoine. Les médias français commencent à s'intéresser au pays lors du blocus de sa reconnaissance par la Grèce (1991). De nombreux articles, commentaires, interviews tentent alors d'éclaircir la situation du pays, mais il $\mathrm{y}$ a eu très peu d'interventions de la part des intellectuels et « experts ». Cette communication montre les grandes lignes des articles parus en France dans la période entre 1991 et 2009. Mais la presse française, en assimilant le peuple macédonien au terme "Slaves », voulait sans doute 
pendant le conflit du Kosovo et le conflit de 2001, éclaircir la situation, mais l'utilisation de ce terme est aussi comprise comme une tentative de négation de l'existence du peuple macédonien. Le nom de la République de Macédoine bloque le pays dans son passé, c'est aux Macédoniens de définir leur identité, pas à leurs voisins ni aux membres de l'UE.

In spite of its famous name, Macedonia is not well-known in France. Till 1991, very few persons even knew the existence of a Republic of Macedonia. The French Medias began to get interested in it with the Greek blocus of Macedonia. A lot of papers, comments, interviews tried then to explain the main lines of the country's situation but with a very light participation of experts or intellectuals. One can find in this paper the main lines of the French press on Macedonia from spring 1999 to September 2001. The French papers, as a whole, assimilating the "Macedonian" people to "Slaves", perhaps to make a clear difference when explaining the situation during the Kossovo crisis or later, during the crisis inside Macedonia, contributed to deny the existence of a "Macedonian" people. The FYROM name is just trapping the country in its past. Macedonians have the right to definite their own identity, not to ask their neighbours or members of the UE!

\section{INDEX}

motsclestr Balkanlar, Yunanistan, Makedonya, Monastir, Bitola

motsclesmk ФРАНЦУСКИОТ ПЕЧАТ (1991-2009), РЕПУБЛИКА МАКЕДОНИЈА (1991-), БАЛКАНОТ, ГРЦИЈА, МАКЕДОНИЈА, БИТОЛА

Thèmes : Histoire

Index chronologique : accord-cadre d'Ohrid (2001), Macédoine -- indépendance, Macédoine (République) -- Histoire (1991-)

Index géographique : Macédoine, Grèce, Balkans, Monastir

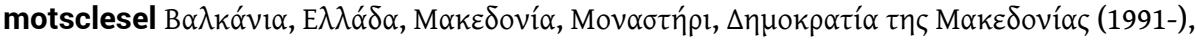

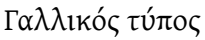

Mots-clés : identité macédonienne

Keywords : Greek/Macedonian Conflict (2001), Greece, Balkans, Monastir, Macedonia -independence, macedonian identity, History, Ohrid Agreement (2003)

\section{AUTEUR}

\section{TONI GLAMCEVSKI}

CREE-CEB EA 4513 